\title{
Development of Reverse Fingerprint Lifting Techniques for Forensic Applications
}

\author{
Keywords \\ Reverse liffing; Microbiome; Developing solution
}

\begin{abstract}
Latent fingerprint lifting has been carried out on objects to identify and classify the human touched objects within a workplace, which can be readily applied for both traditional fingerprint analysis and microbiome-based forensic identification. A total of 20 objects in a work place environment, including cabinet handle, cell phone, door knob, keyboard, mouse, office phone, and stapler, were selected for this study. Four different types of tapes were used for lifting. The lifted invisible prints were then observed by three different methods: 1) forensic light source, 2) powder dusting, and 3) adhesive-side developer. Developed visible fingerprints were then photographed. Forensic light source made the print visible on clear tape and packing tape lifted from several objects including paper weight, and door. Red powder dusting used after lifting was successful with prints on some tapes. Developing solution showed better results with masking tape and packing tape. The results showed that the reverse lifting method worked well with glazed/smooth surfaces such as mug, mouse, window and door, which will allow investigators to use other forensic applications including the microbiome-based analysis as well as the fingerprint lifting on the same object in a crime scene.
\end{abstract}

\section{Introduction}

Fingerprint evidence left behind by a suspect or victim can identify who was in the crime scene and what objects she or he touched. Similarly, it is also possible to identify a suspect from the microbiota collected from the object he or she touched in the crime scene. The question is whether it is possible to collect both microbiome and fingerprint from the same position in the object he or she touched. The traditional method of development of a latent print involves the use of powder or chemical treatment, which clearly destroys the bacterial cells shed by the subject. On the other hand rubbing the surface of the object for collecting microbiome disturb or destroy the fingerprint ridges pattern if present on the object. Therefore the researchers are trying to develop a technique that help collecting both the fingerprint and the microbiome from the same object without destroying either of them.

\section{Background}

Several researches in the field of fingerprint developing techniques have been carried out in the past years. The most widely used visualization techniques for developing latent finger prints are dusting, cyanoacrylate fuming ("superglue"), ninhydrin spray, iodine vaporization, and the use of various fluorescent powders. In all these cases, the applied reagent interacts with the fingerprint deposit to make the ridge detail visible. Subsequent application of dyes or illumination with light of various wavelengths made the print visible. The main drawback in those methods is that the specimen objects

\section{Journal of Forensic Investigation}

\author{
Johnson TC ${ }^{1}$, Brown AS ${ }^{1}$, Oommen $Z^{1}$, Okafor $\mathrm{U}^{1}$, and \\ Lee $\mathrm{Y}-\mathrm{J}^{2}$ \\ ${ }^{1}$ Department of Chemistry and Forensic Science, Albany State \\ University, USA \\ ${ }^{2}$ Department of Biological Sciences, Albany State University, USA
}

*Address for Correspondence

Oommen Z, Department of Chemistry and Forensic Science, Albany State University, Albany, GA 31705, USA; E-mail: zachariah.oommen@asurams.edu

\section{Submission: 06-February-2020 \\ Accepted: 18-March-2020}

Published: 20-March-2020

Copyright: (c) 2020 Johnson TC, et al. This is an open access article distributed under the Creative Commons Attribution License, which permits unrestricted use, distribution, and reproduction in any medium, provided the original work is properly cited.

cannot be used for other forensic evidences like microbiome.

Forensic application of microbiomes have been gaining importance as potential means of associating people with evidence and environments. It has been found that human biota varies systematically across body habitants and time [1]. Several studies have reported to the use of microbiome profile characterization for criminal investigations. The trace microbiome on one's skin and the surface of an object one touches can use as a potential means to identify a person. Humans shed approximately thirty million bacterial cells into their vicinity every hour [2]. Reported that bacterial community found on fingertips could be traced on a key board resulting in the identification of the person who used the keyboard [3]. In another study showed that personal mobile phones carry personal microbial signature of their owners [4,5]. Reported postmortem succession of human associated microbial communities for estimating postmortem interval for forensic analyses [6].

Therefore, researchers turn their attention toward noninvasive fingerprint developing techniques as it does not compromise the sample either for subsequent analysis by other fingerprint enhancement techniques or using the same sample for other forensic evidences. However, published results of research in the noninvasive fingerprint developing field are very limited. Recently, a noninvasive method of visualizing latent fingerprint deposit on thermal paper has been studied and published by John Bond [7]. He has shown that higher intensity UV source gave superior fingerprint visibility compared to other light sources. Also published another noninvasive method to visualizing latent finger prints deposited onto thermal paper by raising the temperature of the paper [8]. In another study, studied laser detection of latent fingerprints on white paper [9]. They measured the UV fluorescence of various kinds of printer-toner and printer-ink printed on high grade white paper and found that the fluorescence intensity of most printed paper is weak, because ink or toner absorbs the fluorescence of the paper. Fourier Transform Infra red imaging was used for noninvasive detection of latent fingerprints [10]. The Infra red images from substrates like trash bags, soda can, 
Citation: Johnson TC, Brown AS, Oommen Z, Okafor U, Lee Y-J. Development of Reverse Fingerprint Lifting Techniques for Forensic Applications. 2020; $8(1): 8$.

ISSN: $2330-0396$

Table 1: Fingerprints on objects lifted by tapes and developed by Adhesive Side developer.

\begin{tabular}{|c|c|c|c|c|c|}
\hline No & Object & Masking Tape & Duct Tape & Packing Tape & Clear Tape \\
\hline 1 & Paper Weight & LPD & LPD & LPD & LPD \\
\hline 2 & Highlighter & LPD & LPD & LPD & LPD \\
\hline 3 & Office Phone & LPD & LPD & LD & LPD \\
\hline 4 & Smooth Wall & LPD & LPD & LD & LPD \\
\hline 5 & Mug (ceramic) & LPD & LPD & LD & LPD \\
\hline 6 & Water Bottle (Plastic) & LD & LD & LPD & LND \\
\hline 7 & Class room table & LPD & LPD & LPD & LND \\
\hline 8 & Cell Phone & LD & LPD & LD & LND \\
\hline 9 & Pencil (Wooden) & LPD & LPD & LPD & LND \\
\hline 10 & Stapler & LPD & LPD & LD & LND \\
\hline 11 & Paper clip holder & LD & LPD & LPD & LND \\
\hline 13 & Writing pen & LPD & LND & LPD & LND \\
\hline 14 & Door & LPD & LND & LPD & LND \\
\hline 15 & Cello tape Dispenser & LPD & LND & LPD & LND \\
\hline 16 & Mouse & LND & LND & LPD & LND \\
\hline 17 & Keyboard & LND & LND & LPD & LND \\
\hline 18 & Door knob & LND & LND & LPD & LND \\
\hline 19 & Computer Screen & LND & LND & LPD & LND \\
\hline 20 & Rough wall & LND & LND & LND & LND \\
\hline
\end{tabular}

LD: Lifted and Developed; LPD: Lifted and Partially Developed; LND: Lifted and Not Developed

Table 2: Shows the results of the reverse lifting done on objects and developed by Red Powder.

\begin{tabular}{|c|c|c|c|c|c|}
\hline No & Object & Masking Tape & Duct Tape & Packing Tape & Clear Tape \\
\hline 1 & Paper Weight & LND & LND & LND & LPD \\
\hline 2 & Highlighter & LND & LND & LPD & LND \\
\hline 3 & Office Phone & LND & LND & LD & LND \\
\hline 4 & Smooth Wall & LND & LND & LND & LND \\
\hline 5 & Mug (ceramic) & LND & LND & LD & LND \\
\hline 6 & Water Bottle Plastic & LND & LND & LND & LND \\
\hline 7 & Class room table & LND & LND & LND & LND \\
\hline 8 & Cell Phone & LND & LND & LPD & LND \\
\hline 9 & Pencil (Wooden) & LND & LND & LND & LND \\
\hline 10 & Stapler & LND & LND & LND & LND \\
\hline 11 & Paper clip Holder & LND & LND & LND & LND \\
\hline 12 & Window (Frame) & LND & LND & LND & LD \\
\hline 13 & Writing pen & LND & LND & LND & LND \\
\hline 14 & Door & LND & LND & LPD & LND \\
\hline 15 & Cello tape Dispenser & LND & LND & LPD & LND \\
\hline 16 & Mouse & LND & LND & LPD & LND \\
\hline 17 & Keyboard & LND & LND & LPD & LND \\
\hline 18 & Door knob & LND & LND & LND & LND \\
\hline 19 & Computer screen & LND & LND & LPD & LPD \\
\hline 20 & Rough Wall & LND & LND & LND & LND \\
\hline
\end{tabular}

LD: Lifted and Developed; LPD: Lifted and Partially Developed; LND: Lifted and Not Developed 
Table 3: Shows the results of the reverse lifting done on objects and viewed by ALS.

\begin{tabular}{|c|c|c|c|c|c|}
\hline No & Object & Masking Tape & Duct Tape & Packing Tape & Clear Tape \\
\hline 1 & Paper Weight & No & No & No & Yes \\
\hline 2 & Smooth Wall & No & No & No & yes \\
\hline 3 & Mug (ceramic) & No & No & No & Yes \\
\hline 4 & Door & No & No & Yes & Yes \\
\hline 5 & Writing Pen & No & No & No & Yes \\
\hline
\end{tabular}

LD: Lifted and Developed; LPD: Lifted and Partially Developed; LND: Lifted and Not Developed

copier paper, cigarette butts are analyzed using ENVI ${ }^{\oplus}$ software (RSI Inc., Boulder, CO). The analysis was further improved by the addition and subtraction of band intensity, addition and subtraction of band intensity. Eric C. Bray published a case report stating the method of developing latent print from duct tape [11]. M.G Campbell in a technical report showed that the use of a hot air blower can separate adhesive tapes and permit a more efficient lifting from cardboard surfaces [12-14].

Traditional methods of fingerprint development are invasive as the use of powder or chemical treatment on the objects destroy the other forensic evidences. Fingerprint powders can contaminate the evidence as well as environment especially in an office set up. In this context we are proposing a non-invasive method, the reverse fingerprint lifting techniques, in which there is no powder or chemical treatment to the object in question, is favored as it does not compromise the object for other forensic evidences like microbiome. This research concentrate on reverse lifting method of fingerprint development where tapes are used to lift the latent print from objects and areas that prints are suspected. The technique is noninvasive and is based on how well the oils and sweat that creates a fingerprint are able to transfer from the object to the adhesive side of the tape. Powder or chemical treatment would then be applied to the adhesive side of the tape. This approach will avoid dusting or chemically treating objects directly and will allow the workplace to remain clean and healthy after fingerprint collection.

The research is to select suitable objects, that can be successfully employed for both finger print and microbiome based forensic identification. The latent print collected from such objects can be compared with known fingerprints collected from the selected subjects either with ink or digitally with live scan devices. Multiple enhancement techniques along with alternate light source (forensic light source) can be used.

\section{Materials and Methods}

\section{Objects}

The method involves collection and processing, which means lifting the latent print from the source and then processing to develop the print. This method is just opposite of traditional invasive fingerprint development called reverse lifting. 20 objects that are usually found in an office set up were selected to carry out the experiment. They are computer screen, paper weight, glass window, computer key board, computer mouse, cell phone, door knob, door, writing pen, mug, paper clip holder, wall (smooth surface) wall (rough surface), water bottle, office phone, highlighter, table, wooden
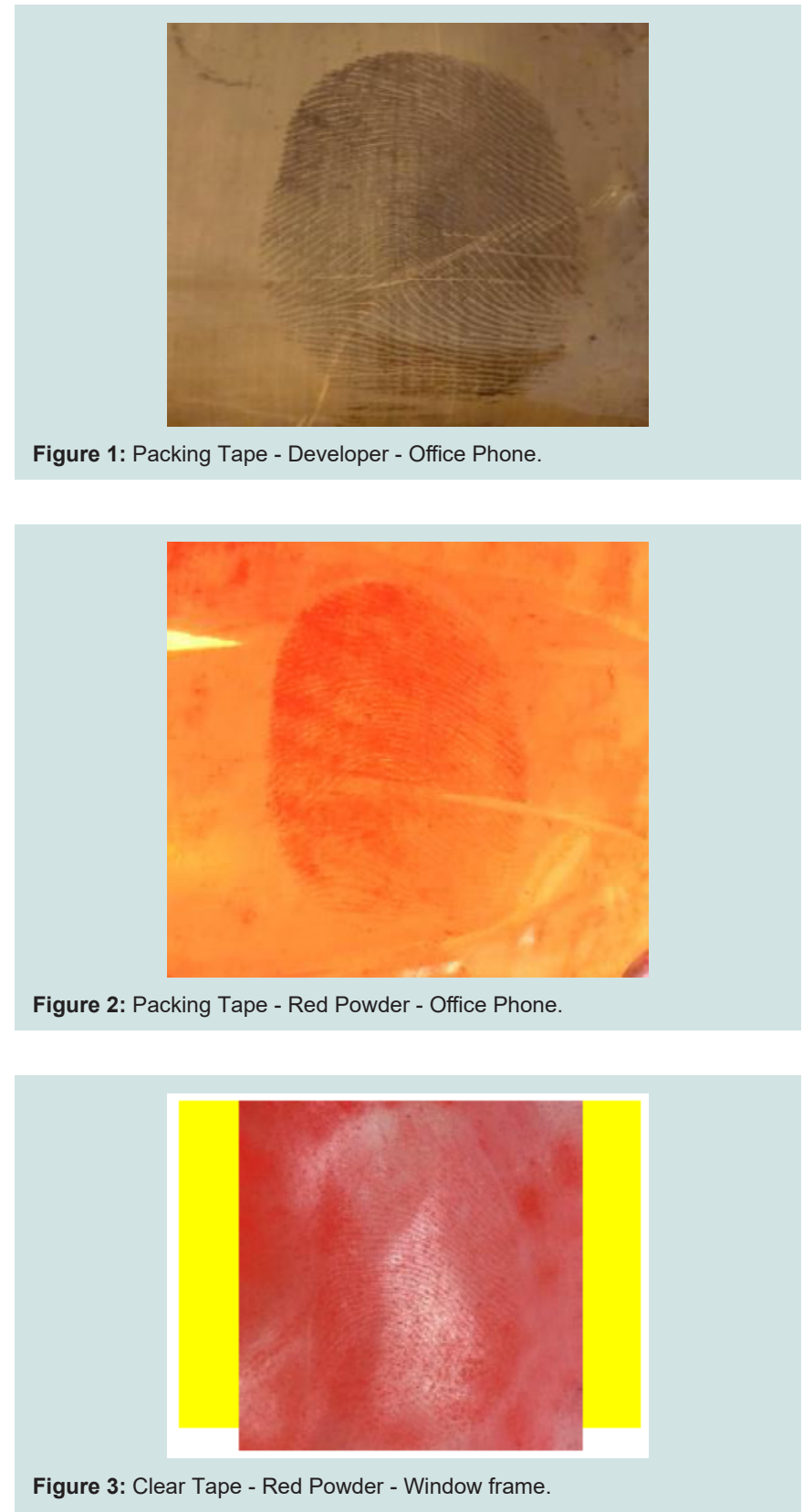

pencil, stapler, and cabinet handle. Red/black print powder, magnetic powder, dusting brush, illuminator, and adhesive-side developer were used to develop the latent prints.

A forensic light source, Luma-Lite R2000 A, (Dayton Scientific Inc) at wavelengths $365,450,485$, and $570 \mathrm{~nm}$ is used for enhancing the visualization. The four different types of tapes that were used in this experiment were making tape, packing tape, clear tape and duct tape. Each tape produced different results. The objects and the developed prints from the tapes are photographed documented by a Canon EDS REBEL T5i

\section{Deposition of the fingerprint}

Intentional latent fingerprints were placed on each of the 20 objects by placing a finger onto the selected objects for 1-2 seconds 
Citation: Johnson TC, Brown AS, Oommen Z, Okafor U, Lee Y-J. Development of Reverse Fingerprint Lifting Techniques for Forensic Applications. 2020; 8(1): 8.
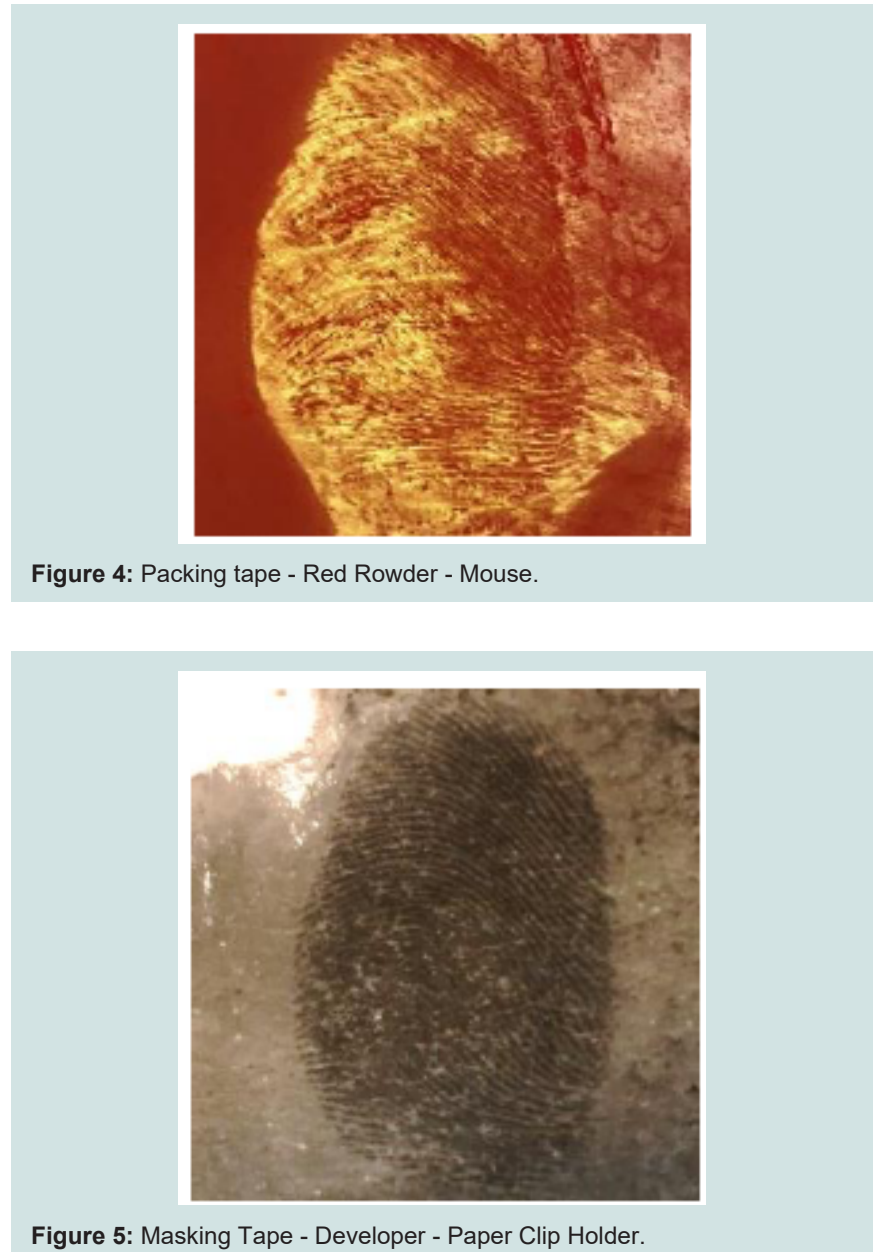

Figure 5: Masking Tape - Developer - Paper Clip Holder

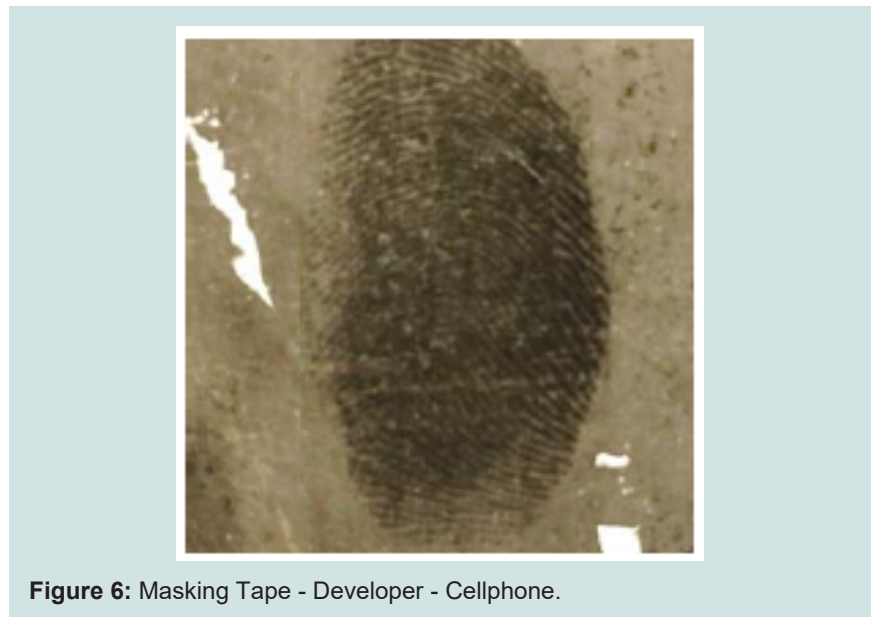

with light pressure sufficient to ensure the contact between the finger and the object. Attention is made to keep the time and pressure same to all objects to maintain uniform deposition.

\section{Lifting of the fingerprint}

To detect and enhance the fingerprint on the surface, a forensic light source was used. After the fingerprint is illuminated, if possible, a photograph of the print is taken. Then the print was lifted with
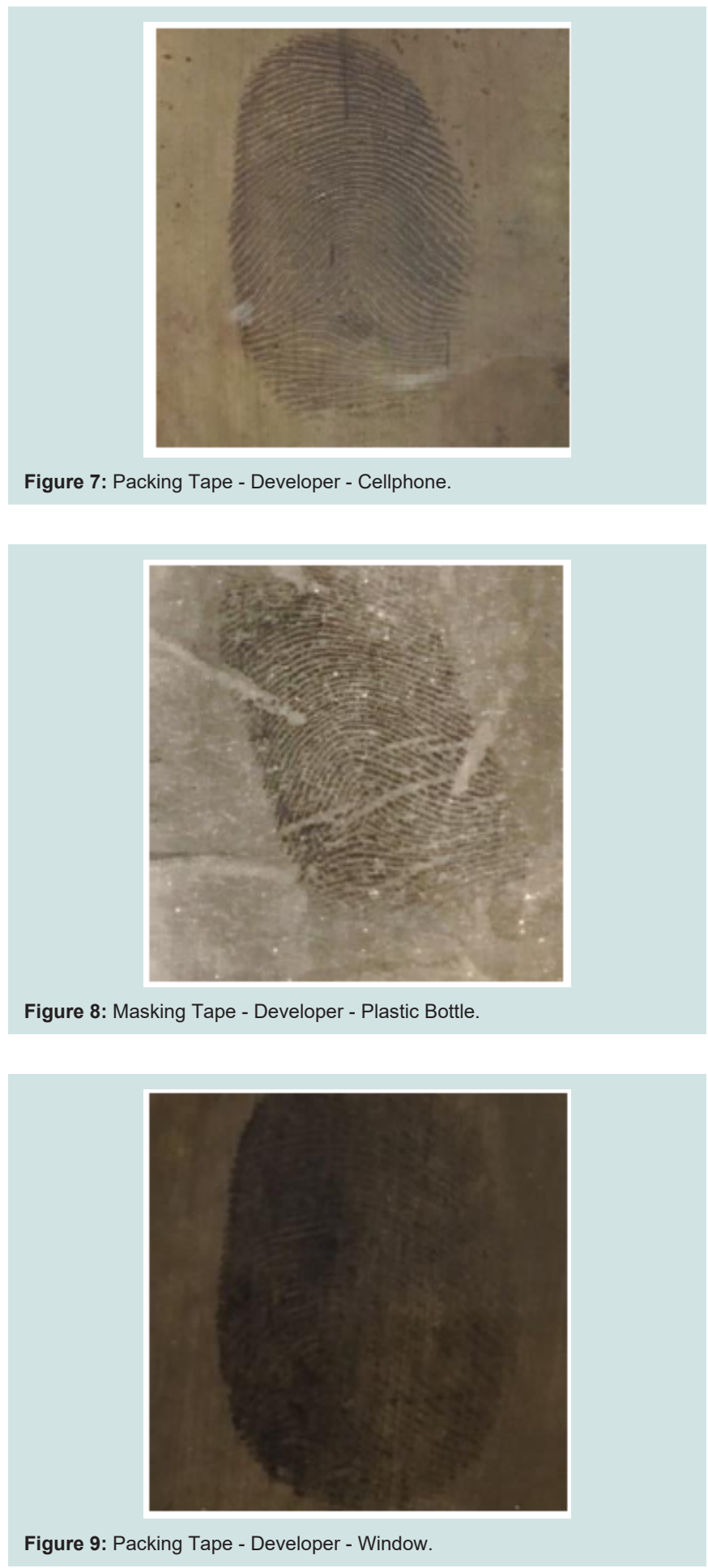

packing tape, duct tape, masking tape and clear tape. The tapes were then examined under the alternative light source and photographed.

\section{Developing the fingerprint with Light Source}

The lifted print on the tapes which are not visible to the naked eye, require treatment to cause them visible. In this technique used an alternative light source to make the print 

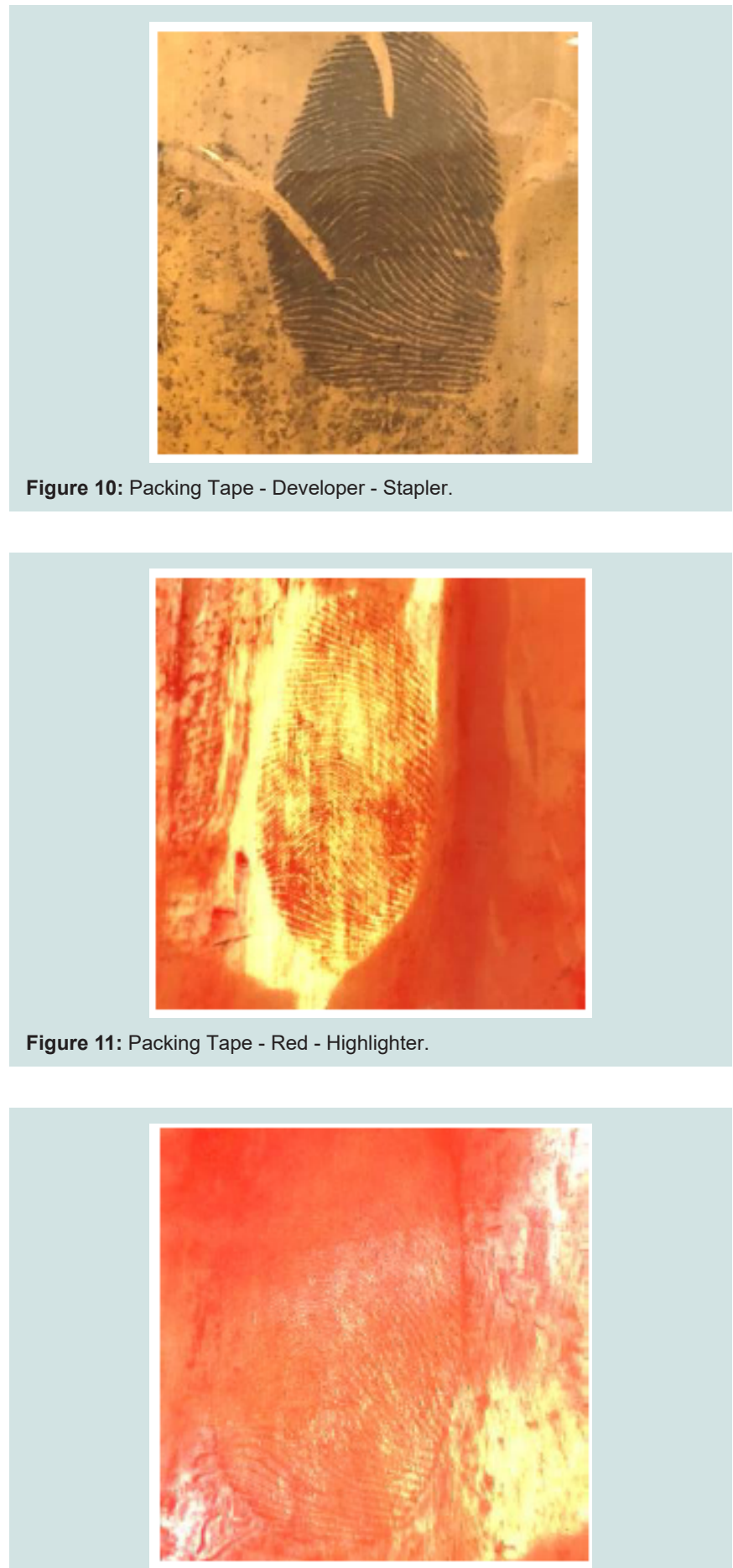

Figure 12: Packing Tape - Red Powder - Computer Screen.

visible. Then developed the latent print by the application of both powder and adhesive side developers. For this the tape was laid on a surface in a rotating manner. The developer was swabbed over the surface while rotating the application. Developing the fingerprint with Powder Dusting

Fingerprint powder is applied to the surface of the tapes bearing the latent print with a fingerprint brush.
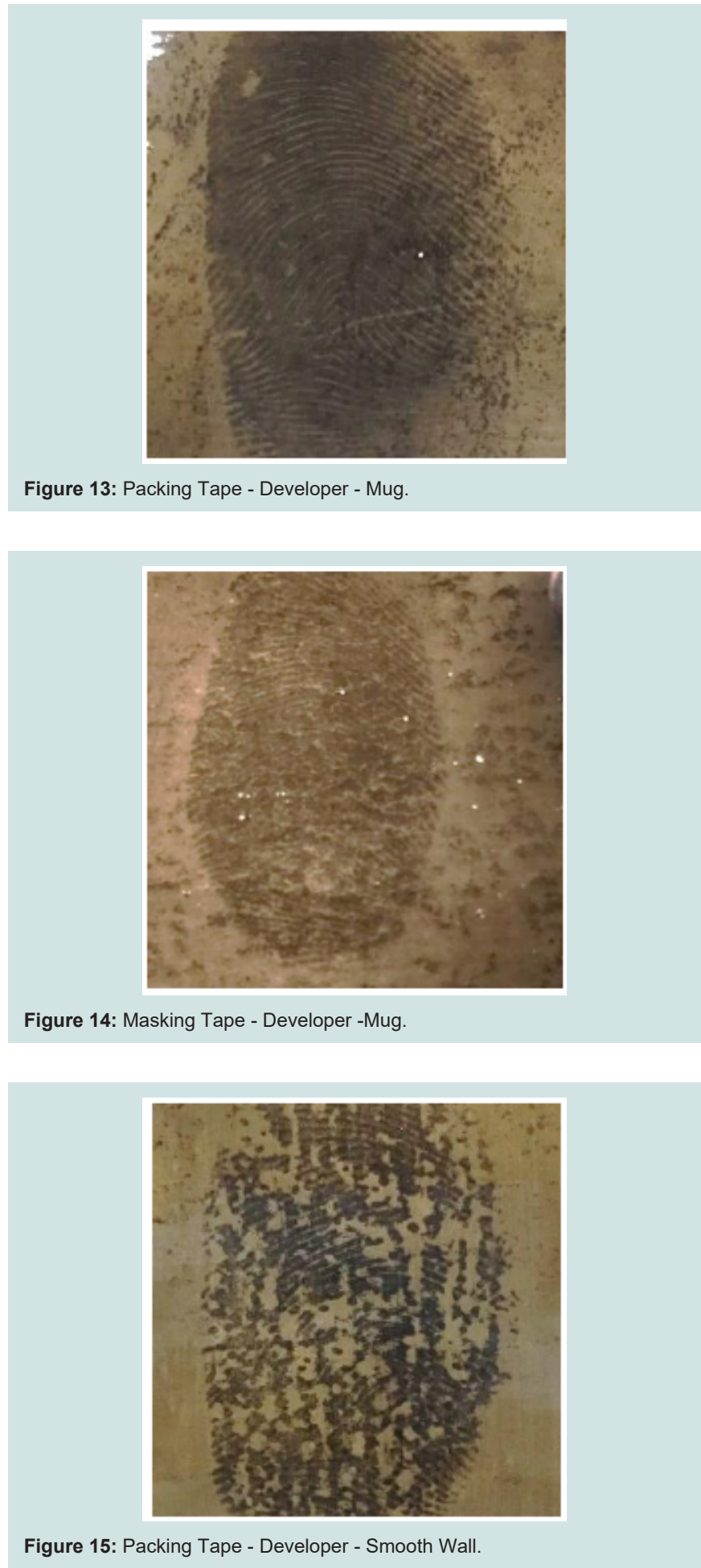

Developing the Fingerprint with Adhesiveside Developer

First the brush is dipped gently into the developer solution and then slightly swirl over the latent print area in the tapes for 30 to 40 seconds. The adhesive side solutions adhere to the area in the form of a print. The developer was washed away with water to reveal the print. A photograph was then taken. 


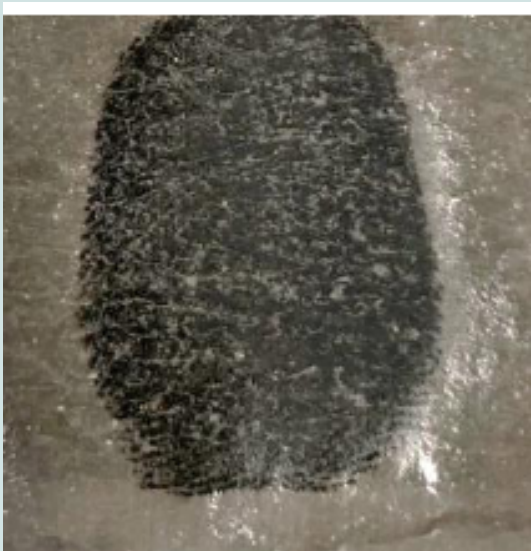

Figure 16: Masking Tape - Developer - Office Phone.

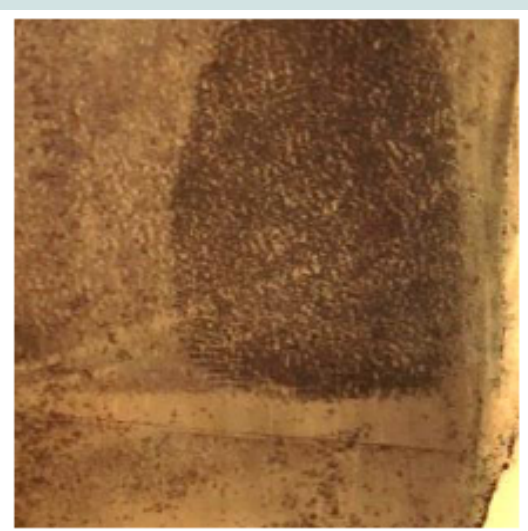

Figure 17: Packing Tape - Developer - Tape Dispenser.

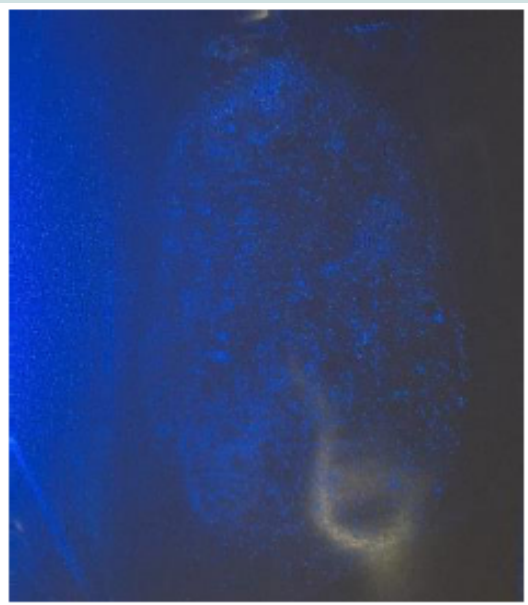

Figure 18: Clear Tape ALS - Paper Weight.

\section{Results}

Fingerprints on objects lifted by the tapes and developed by

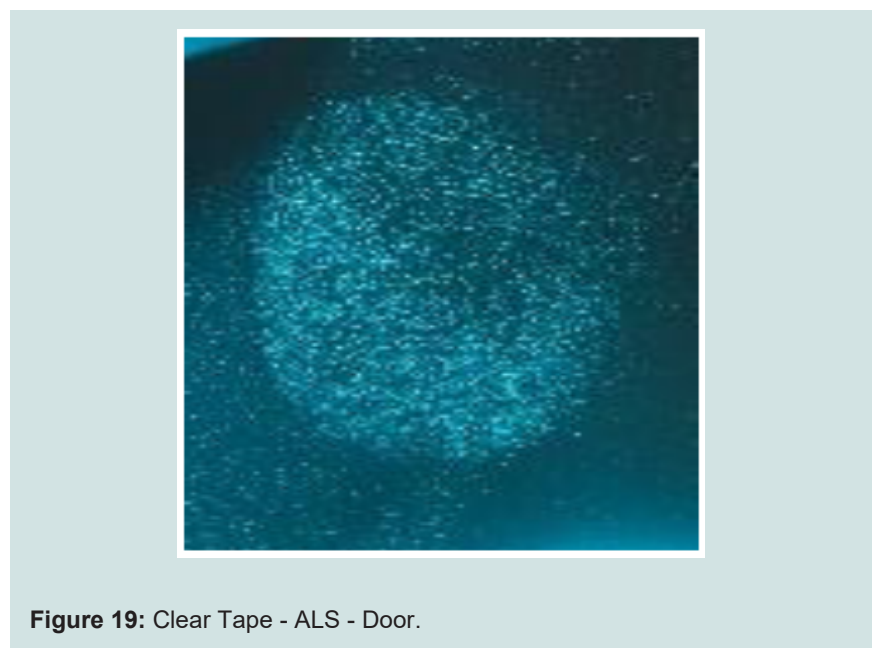

\section{Adhesive Side Developer}

Almost all the fingerprints placed on the objects are invisible to the naked eye but some are visible under the Alternate light Source (ALS). Prints are lifted by using masking tape, duct tape, packing tape, and clear tape and then developed using chemical developer. Table 1 shows the results of the reverse lifting done on objects and developed by adhesive side developer.

Adhesive side developer method was successful in developing prints on all four tapes in the case of From plastic water bottle, class room table, cell phone, pencil, stapler and paper clip holder, the fingerprints were able to lift the print using three tapes. Fingerprints from all the rest objects were lifted by only one type of tape. All the prints made on the objects were invisible to the naked eye but some were visible under Alternate Light Source (ALS). Prints are lifted by using masking tape, duct tape, packing tape, and clear tape and then developed using chemical developer. Experiments showed that the quality of the fingerprint depends on the object's surface and the type of tape used.

\section{Fingerprints on objects lifted by the tapes and developed by Red Powder}

Of the twenty objects,only 6 developed ridges with red powder and only a very few objects produced well defined ridges like office phone, mug, window frame.

Objects lifted by the tapes and viewed by Alternate Light Source (ALS)

Almost all the fingerprints placed on the objects are invisible to the naked eye but some are visible under Alternate light Source (ALS). Table 3 shows the results of the reverse lifting done on objects and viewed by ALS

\section{Discussion}

The research mainly deals with quality of the latent prints developed in the reverselifting method. Latent prints developed are graded based on the quality of ridge details visible on the tapes. The grading is numbered from no development (zero) to fully developed (4) based on the grading by Bandey et.al $[13,14]$. Fingerprint ridge development on different tapes after the 
application of adhesive developer solution was graded based on the quality of ridge detail visible on the tapes. The quality of the print is expressed by numbers as developed by Bandey $[13,14]$.

\begin{tabular}{|c|c|}
\hline Grade & Comments \\
\hline 0 & No development \\
\hline 1 & No continuous ridges, All discontinuous or dotty \\
\hline 2 & One-third of mark continuous ridges (rest no development, dotty) \\
\hline 3 & Two-thirds of mark continuous ridges (rest no development, dotty) \\
\hline 4 & Full Development, Continuous ridges \\
\hline
\end{tabular}

Of the 20 objects only 7 succedded to produce well defined fingerprint ridges in the grade 4 category (Figures 1-10). Some are partially developed as shown in the (Figures 11-13). Some other cases show the shape of the print with no clear ridge details (Figures 14-17). Moreover some fingerprints are visible in Alternate light source before development (Figures 18 and 19). No print was able to developed from rough wall. Packing tape appears to be more effacing in producing ridge details of category [4]. The lifting and developing efficiency of the tapes decrease in the order packing tape, masking tape, duct tape and clear tape. Developing agent also influences the ridge details as it is evident from the figures that developer solution works better on most of the 9 objects rather than red powder.

Based on the results it is very clear that the developed print depends on several factors. Quality of the print depends on the surface of the objects that are touched, condition surrounding the contact between friction skin and the objects, and also the amount and type of residue on the skin. It is also based on the substrates on which the print is transferred. Porous substrates are generally absorbent and the prints deposited into these media absorb into the substrate and are somewhat durable. Nonporous surface does not absorb. These surfaces repel moisture and often appear polished. They include glass, metal, plastic, lacquered or polished wood, rubber etc. Latent prints on these substrates are more susceptible to damage because the fingerprint residue resides on the outermost surface. Semi-porous surfaces are characterized by their nature to both resist and absorb fingerprint residue. Fingerprint residue on these surfaces may or may not soak in because of the absorbent properties of the substance and the variable viscous properties of the fingerprint residue. These surfaces include glossy cardboard, glossy magazine covers, some finished wood, and some cell phones. Latent print secretions are complex emulsifications of numerous and varying compounds. It can be water soluble consisting of sodium chloride and amino acids (Serine and glycine) or water insoluble non-polar lipids and proteins [15].

Identifiable ridge details of grade 4 are obtained on objects like Office phone (Figures 1,2), window frame (Figure 3), Mouse (Figure 4), Paper clip (Figure 5), Cell phone (Figure 6 and 7), Plastic Bottle (Figure 8), and Stapler (Figure 10). All the objects of these types have non-pours and polished surface. The finger print residue resides on the outermost surface and can be easily lifted by the tapes. The ridge details are continuous and all the minutiae points are easily located. Packing tape appears to be a good lifter among the four types. Packing tape is not a good choice for Plastic bottle and paperclip holder. Masking tape is good for lifting prints from a plastic bottle.

Grade 3 types are obtained for objects like highlighter (Figure
11), Computer Screen and Mug are not fully developed in all areas of the print (Figure 12 and 13). Only two-thirds of the ridges are continuous, the rest are discontinuous or dotty. For cell phone duct tape and developer solution are used. Office phone print is lifted by packing tape but developed by red powder.

Grade 2 types where one-third of the ridge details are visible, are obtained for smooth wall (Figure 15), office phone and tape dispenser (Figure 16 and 17). Those prints only show the shape of the finger print but not any of the ridge details.

All the rest of the pictures are either not developed or dotty. No information can be obtained from these types of ridges and belong to grade 1 .

\section{Effects of tapes on lifting}

Masking tape: Fingerprint development on the adhesive side of masking tape using adhesive side developer have been mostly successful. The prints are mostly clustered in the middle but ridge detail is clear on the outer part of the print (Figures 5,6,8 and 14). Viewing the print under the light source and with the naked eye was not successful. Developing with red powder has also been unsuccessful.

Duct tape: Duct tape has the least fingerprint visibility. The print is more visible around the outer part. Duct tape only works with adhesive-side developer and was unsuccessful under the light source, with powder and with the naked eye.

Packing tape: Fingerprints collected using packing tape shows clear ridge details. It works best with adhesive-side developer and with red powder for most of the objects (Figures 1,2,4 and 7).

Clear tape: The clear fingerprint lifting tape was specifically designed to lift fingerprints that will later be used in evidence. Most of the prints were not developed either with developing solution or red powder. Clear tape was successful to ALS in a few cases (Figures 18 and 19).

\section{Conclusion}

Reverse lifting methods for fingerprint development has been successfully performed in at least seven objects out of 20 objects selected for the study. The results have shown that the ridge details developed in the new methods are comparable to the conventional methods of fingerprint development. The objects selected for the study from an office setup can be very well used for other forensic evidence examination as the method does not contaminate or destroy other forensic evidences like microbiome. Cell phone, office phone, mouse, mug, platic water bottle, paperclip holder etc are some of the objects successfully tested for reverse lifting. Packing tape and masking tape were able to lift the invisible print more successfully compared duct tape and clear tape. Developing solution worked well with those objects compared to powders. Other chemical methods of development are under consideration for future studies.

\section{References}

1. Elizabeth KC, Christian LL, Micah H, Noah F, Jeffrey IG, et al. (2009) Baterial community variation in human body habitants across space and time. Science 326: 1694-1697.

2. Qian J, Hospodsky D, Yamamoto N, Nazaroff WW, Peccia J (2012) Size resolved emission rates of airborne bacteria and fungi in an occupied 
Citation: Johnson TC, Brown AS, Oommen Z, Okafor U, Lee Y-J. Development of Reverse Fingerprint Lifting Techniques for Forensic Applications. 2020; $8(1): 8$.

classroom. Indoor Air 22: 339-351.

3. Fierer N, lauber CL, Zhou N, McDonald D, Costello EK, et al. (2010) Forensic identification using skin bacterial communities. Pro Natl Acad Sci U S A 107: 6477-6481.

4. Meadow JF, Altrichter AE, Green JL (2014) Mobile phones carry the personal microbiome of their owners. PeerJ 2: e447.

5. Lax S, Hampton-Marcell JT, Gibbons SM, Colares GB, Smith D, et al. (2015) Forensic Analysis of microbiome of phones and shoes. Microbiome 3: 21.

6. Kathleen AH, Kelly LC, Lee MJ, Tim ES, Jennifer MB (2015) Estimating time since death from postmortem human gut microbial communities. J Forensic Sci 60: 1234-1240.

7. Bond JW (2015) A noninvasive and speculative method of visualizing laten fingerprint deposits on Thermal paper. J Fore Sci 60: 1034-1039.

8. Bond JW (2013) Development of latent Fingerprints on thermal paper by controlled application of heat. J Fore Sci 58: 767-771.

9. Norimitsu A, Naoki S K, Kuroki, Kenji K (2011) Visualizing latent fingerprints on colorprinted papers using ultraviolet fluorescence. J Fore Sci 56: 754-759.

10. Nicole JC, Edward GK, Rebecca SP, Scott H (2007) Infrared Spectroscopic Imaging for Noninvasive Detection of Latent Fingerprints. J Fore Sci 52: 4853.

11. Berg EC (1992) Latent prints from duct tape. J Fore Identification 42: 401 403.

12. Campbell BM (1991) Separation of adhesive tapes. J Fore Identification 41: 102-106.

13. Bandey HL (2004) The Powder Process, Study 1: Evaluation of Fingerprint Brushes for Use with Aluminum Powder; HOPSDB Report. Home Office Police Scientific Development Branch Sandridge, U.K 54.

14. Bandey HL, Kent T (2003) Superglue Treatment of Crime Scenes-A Trial of the Effectiveness of the Mason Vactron SUPERfume Process. HOPSDB Report. Home Office Police Scientific Development Branch: Sandridge, U.K 30.

15. Bleay S, Cantu A, Inlow V, Ramotowski R, Sears V, et al. Latent print development Brian Yamashita and mike French pp: 1-68.

\section{Acknowledgement}

This research was supported by Award No. 2016-DN-BX-0196, awarded by the National Institute of Justice, Office of Justice Programs, U.S. Department of Justice. The opinions, findings, and conclusions or recommendations expressed in this publication are those of the author(s) and do not necessarily reflect those of the Department of Justice. 